\title{
Component representation for shock qualified foundation structure
}

\author{
Timothy Coats ${ }^{\mathrm{a}, *}$, Michael Cooper $^{\mathrm{a}}$, Kevin $_{\text {Arden }}^{\mathrm{a}}$ and Fred Russell ${ }^{\mathrm{b}}$ \\ ${ }^{a}$ Newport News Shipbuilding, 4101 Washington Avenue, Newport News, VA 23607, USA \\ ${ }^{\mathrm{b}}$ General Dynamics Electric Boat 75 Eastern Point Road Groton, CT 06340, USA
}

Received 7 December 2001

Revised 23 July 2002

\begin{abstract}
Navy ship foundations are typically designed for shock using the Navy's Dynamic Design Analysis Method. The NAVSEA 0908-LP-000-3010, Rev. 1 manual states that a component modeled as a lumped mass with rigid links should not provide constraint to the support structure. This ensures foundations are designed to withstand all shock loads without accounting for additional stiffness provided by the component. Investigations are provided herein to illustrate the trade-offs and consequences of several approaches for component representations. The observations reinforce the notion that one must give careful consideration for the system being modeled, the expected modal characteristics, and compliance with NAVSEA 0908-LP-000-3010, Rev. 1.
\end{abstract}

\section{Introduction to foundation shock analysis}

Dynamic Design Analysis Method (DDAM) is the Navy's analytical method for shock qualification for components that are too large for practical testing and for foundations. The DDAM methodology is presented in NAVSEA 0908-LP-000-3010, Rev.1 (referred to herein as the 3010 manual). The methodology is founded upon dynamic analysis procedures developed by the Naval Research Laboratory in the early 1960's.

In ship design, DDAM implemented within a finite element analysis (FEA) platform is a standard tool for sizing foundation structure. Finite elements are used to analytically describe the structure or component, obtain the natural frequencies, and compute the stresses and deflections. Once the results have been obtained, the structure is evaluated to determine if the computed stresses and deflections satisfy the acceptance criteria given for its location and level of shock loading [1].

\footnotetext{
*Corresponding author. Tel.: +1 757380 3128; E-mail: coats tw@nns.com.
}

\section{Current analysis methodology}

The component analysis may use a fixed-base assumption and completely ignore foundation stiffness and modal characteristics. Likewise, the foundation analysis may use a fixed-base assumption where the component is represented as a concentrated mass at the component center of gravity (c.g.) and is linked to the foundation structure; component stiffness and local modal characteristics are ignored. This approach allows the foundation designer and component designer to work independently and is generally the most convenient approach for components that are shock qualified by test. Exceptions to this approach sometimes are needed where the foundation and the component are both qualified by analysis.

Where shock analysis of foundation structure is concerned, careful consideration is required for appropriate component representation. Consider a typical 'tabletop' configuration such as shown in Fig. 1. Historically, this system has typically been modeled as a concentrated mass at the component c.g. linked to a configuration of beams and plates using infinitely rigid links. Where the links attach to the foundation, the nodal rotations are usually free. If the nodal degrees of freedom at the 


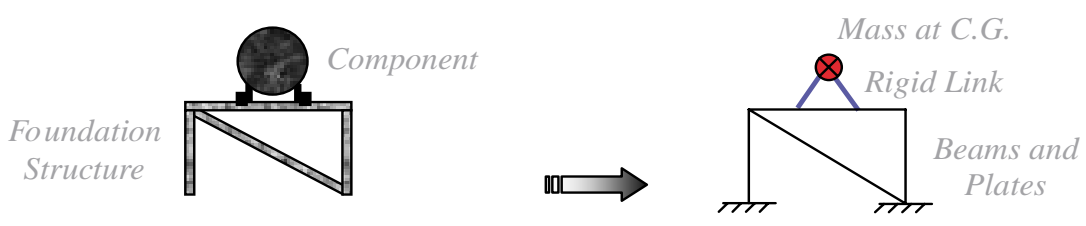

Fig. 1. Typical shock analysis model for DDAM.

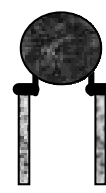

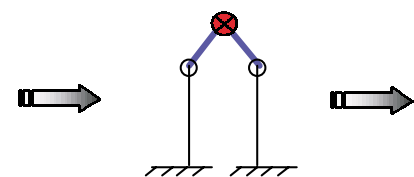

- Realistic and acceptable

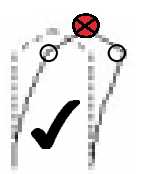

Bending Mode

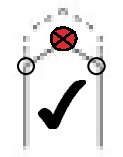

Extension/ Compression Mode

$\mathrm{X}$ - Unrealistic and damaging, but compliant with 3010

Fig. 2. Atypical symmetric model for DDAM.

component c.g. prevent the links from rotating or translating relative to each other, then the rigid link configuration in Fig. 1 is equivalent to a rigid frame pinned at the foundation. This actually provides constraint to the foundation structure.

The pinned rigid frame is a potential violation of a fundamental principle of foundation design; "Where an item is modeled as a lumped mass with rigid links, the equipment model should not provide constraint to the support structure" (Section 3.2.6 of the 3010 manual [1]). However, if the links are pinned at the component c.g. and the foundation, resulting modes may create loads that stretch and pry foundation structure; this may be unrealistic behavior depending on the system configuration. For example, consider the atypical system configuration in Fig. 2; a component bolted to two cantilevers with identical cross-sections. For the configuration in Fig. 2, the structural behavior of the system should comply with section 3.2.6 of the 3010 manual, and the component representation should not produce unrealistic and damaging modes such as prying. Symmetric bending and extensional modes are realistic and acceptable.

Another general practice in foundation design is foundation symmetry. If the cantilevers in Fig. 3 are not equally stiff, the foundation is asymmetric and transmits unbalanced loads into the component. This is problematic when the component is analyzed independently with a fixed-base that does not account for these unbalanced forces. Resistance to deflection in the vertical direction is unbalanced causing a rocking or tilting mode, and should be avoided [1]. However, whether asymmetry can or cannot be avoided, compliance with section 3.2.6 of the 3010 manual is still required. In generally complex structures, some degree of structural asymmetry will likely exist.

Compliance with the 3010 manual is demonstrated by the first two modes shown in Fig. 3 and are marked with a $\sqrt{ }$. The independent bending and rocking/tilting modes occur because the component attachment does not transfer load from one cantilever to the other. The bending and extension are proportionate to the beam section properties. If the component attachment constrains the support structure and therefore transfers load from one beam to the other, a constrained bending mode occurs that does not comply with the 3010 manual. Finally, the prying mode complies with the 3010 manual but is unrealistic and potentially damaging to the structure. The characteristics of a system modeled with an appropriate component representation can be summarized as the following:

- The component attachment should not overly constrain the foundation.

- The component model should not introduce fictitious and damaging responses.

\section{Purpose and objectives}

The purpose of this effort is to compare and contrast various lumped-mass component representations. The objectives of this study are:

- Increase awareness of component representations used in shock analysis performed by DDAM. 


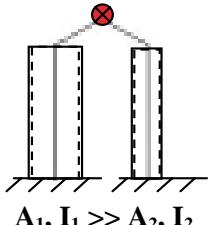

$\mathbf{A}_{1}, \mathbf{I}_{1} \gg \mathbf{A}_{2}, \mathbf{I}_{2}$

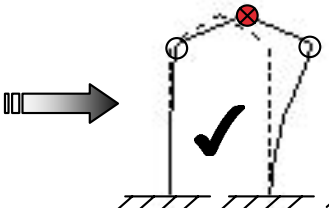

Independent Bending

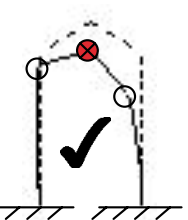

Rocking/ Tilting

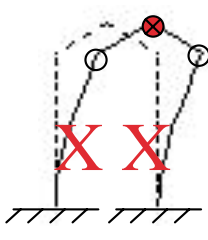

Constrained

Bending

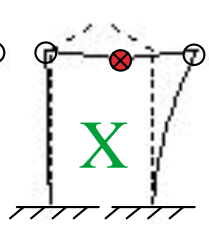

Prying

$\checkmark$ - Realistic and acceptable XX - Non-compliant with $3010 \quad \begin{array}{r}\text { X - Unrealistic and damaging, } \\ \text { but compliant with } 3010\end{array}$

Fig. 3. Atypical asymmetric model for DDAM.

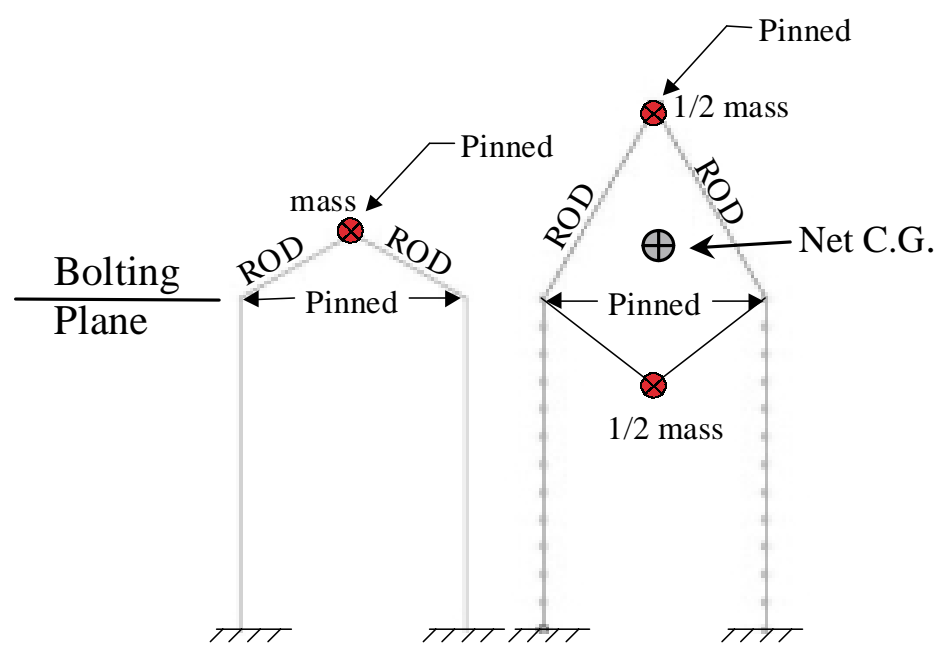

Fig. 4. Rod cases (single and double mass).

- Develop a summary of important characteristics for engineers to use in determining the most appropriate lumped-mass component representation.

- Resolve modeling issues and expedite structural optimization.

\section{Analytical approach}

The approach used to achieve the stated goals was to develop a series of small test cases and observe their behavior under static and shock loading. Hull mounted, full elastic shock inputs were used. All analyses were run using the NASTRAN [2] and ABAQUS [3] structural analysis codes. The test case models shared the following characteristics:

- Two dimensional models, in-plane action only.

- Beam cross sections (structural tubing) were either $4 " \times 4 " \times 1 / 4$ " or $6 " \times 6 " \times 1 / 2$ ".

- The beam cantilevers were 72 inches tall and were set 48 inches apart on centerline.
A component was represented by a concentrated mass located at a net center-of-gravity on the vertical plane of symmetry at a 30-degree angle from the top of the foundation. The mass was linked to the structure using the various concepts described below. The beam structure added no mass.

\section{1) Rod elements (Rod case)}

Two test cases using rod elements are shown in Fig. 4. Rod elements are tension-compression elements with no bending capability. The rod ends are pinned at the component C.G. and at the foundation, and as such do not constrain the structure nor do they distribute mass.

Because the ends are pinned, loads are transmitted along the element's axis. Vertical motion or loading of the mass point produces lateral loads and motion in the foundation (i.e., prying). In an attempt to reduce the magnitude of prying, the mass was split into two parts so that the net c.g. remained the same, but the actual mass points were above and below the bolting plane. 
Table 1

Summary of static analysis results

\begin{tabular}{|c|c|c|c|c|c|c|c|}
\hline Case & Load transfer & Static reaction forces & & & & & \\
\hline & & Left leg & & & Right leg & & \\
\hline & & Fx,lb & Fy, lb & $\mathrm{Mz}$, in-lb & $\mathrm{Fx}, \mathrm{lb}$ & Fy, lb & $\mathrm{Mz}$, in-lb \\
\hline Single mass-rod & No & 0 & 0 & 0 & $-1,000$ & 0 & 72,000 \\
\hline Double mass-rod & No & 0 & 0 & 0 & $-1,000$ & 0 & 72,000 \\
\hline Rigid & Yes & -500 & 0 & 36,000 & -500 & 0 & 36,000 \\
\hline Combination & Yes & -500 & 0 & 36,000 & -500 & 0 & 36,000 \\
\hline Interpolation & No & 0 & 0 & 0 & $-1,000$ & 0 & 72,000 \\
\hline
\end{tabular}

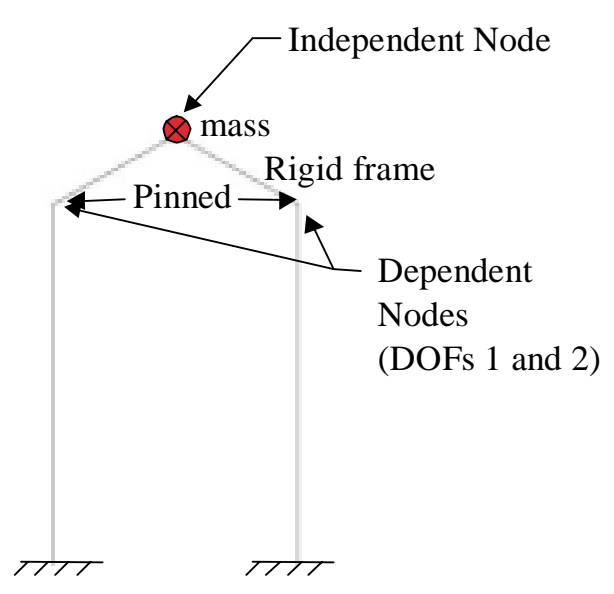

Fig. 5. Rigid case.

\section{2) Rigid frame (Rigid case)}

The pinned rigid frame model in Fig. 5 connects the mass point to the foundation's translational degreesof-freedom only; there were no rotational degreesof-freedom connected. This is referred to herein as a pinned rigid frame because the links do not rotate relative to each other. The NASTRAN RBE2 and the ABAQUS Kinematic Coupling elements were used in the analyses for this component representation. These elements are similar to Multi-Point Constraints (MPC's), but offer the utility of relating the motion of a number of dependent nodes (versus just one) to that of a single independent node. They are enforced in both NASTRAN and ABAQUS through the elimination of the specified degrees of freedom at the dependent nodes.

\section{3) Interpolation method (RBE3 case)}

The interpolation method provides a means to distribute the component mass to the foundation without enforcing a rigid connection between the legs; the mass distribution does not introduce additional dynamic degrees-of-freedom into the problem. The mass el-

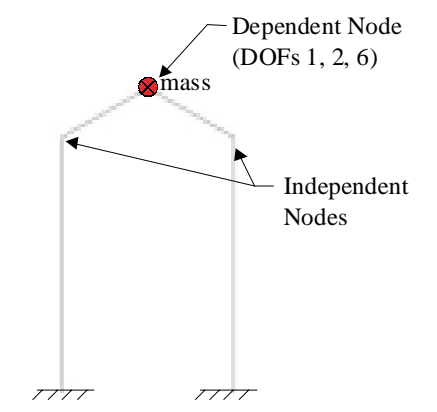

Fig. 6. Interpolation (RBE3) case.

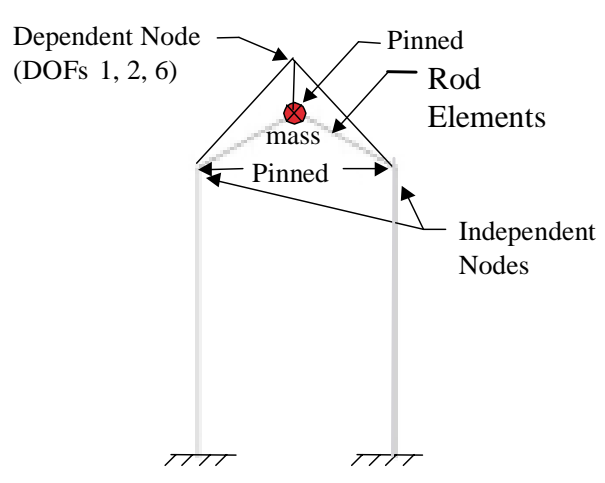

Fig. 7. Combination (combo) case.

ement in Fig. 6 is associated with the dependent node and is distributed to the independent nodes. The mass distribution takes into consideration the location of the dependent node relative to the independent nodes. This provides for the proper calculation of forces due to overturning. The NASTRAN RBE3 and the ABAQUS Distributing Coupling elements were used in the analyses for this component representation. These elements can be conceptualized as flexible implementations of MPC's and the RBE2 and Kinematic Coupling elements described above. 


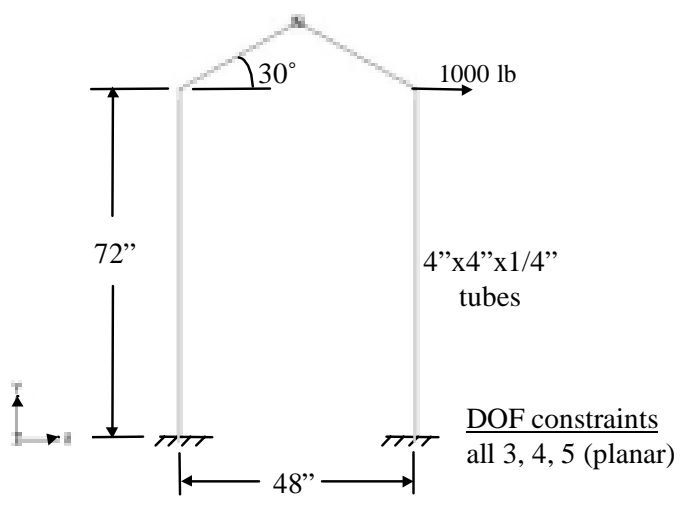

Fig. 8. Static analysis model.

\section{4) Combination of the rod elements and the interpolation method (Combo case)}

An approach was developed that combined the connecting properties of rods and the averaging property of the interpolation method. This model is referred to as the combination model. The mass is connected to the structure and the dependent node using rods. The dependent nodal displacements of the interpolation method force the mass node to displace approximately as the average of the independent nodal displacements. This prevents the vertical displacement of the mass node from prying apart the two cantilevers.

\section{Static analysis results}

The static analysis model in Fig. 8 is useful for illustrating structural constraint by the component representation. This figure does not show all of the component representations. It is, however, representative of the boundary and loading conditions applied in all cases. A 1,000 pound load was applied to the right cantilever for all component representation cases as shown in Fig. 8. Reaction forces computed at the fixed base are provided in Table 1; the corresponding deformation plots are shown in Fig. 9. These deformations and forces show that the Rod and RBE3 cases do not allow load transfer through the component, i.e., the component does not provide constraint to the support structure. However, load is transferred through the component for the Rigid and Combo cases, therefore violating the constraint principle in the 3010 manual. The undeformed configuration is shown with the deformed configuration for reference.

\section{Shock analysis results}

The shock analysis models are shown in Fig. 10. This figure does not show all of the component representations. It is, however, representative of the boundary conditions and the structural and mass configurations in all cases. The asymmetric structure and mass configurations were selected to illustrate the differences in response for the component representations considered. The 3010 manual strongly recommends avoiding asymmetry, however, asymmetry is often unavoidable in actual ship configurations.

A summary of the results for the symmetric structure and mass model is provided in Tables 2 and 3. Reaction forces were calculated and are dependent on frequency, mass participation, and surface ship full elastic, hull mounted shock response spectra. The ' $\mathrm{V}$ ' and ' $\mathrm{B}$ ' in the mass participation column represent 'vertical' and 'bending' modes, respectively. A ' $20 \mathrm{~V}$ ' in the mass participation column indicates that the mode is primarily a vertical mode with a $20 \%$ mass participation and the resulting reaction forces are computed using vertical shock motion inputs. Corresponding mode shapes are provided in Fig. 11.

The mode shapes and reaction forces were investigated and the following observations are made:

- Since the rod forces acted along the axis of the rods and both beams that comprise the foundation were weak in bending, the vertical response mode was characterized by an extreme and unrealistic prying apart of the two beams. The lateral reaction forces and moments due to this prying action were much greater than the other cases. Vertical reaction forces were much lower than for the other cases because of a significant lowering of the response frequency. (DDAM modal forces are a function of frequency, modal mass, mass participation and mode shape.)

- The double-mass rod case introduced two additional modes. Had these masses been symmetrically located with respect to the bolting plane and the modeled foundation was itself symmetric, two of these four modes would have had zero modal mass and would not have been selected. When a lack of symmetry is introduced (in this case by moving the net center of gravity above the bolting plane), these additional modes gain modal mass and may be selected. In this case, both vertical modes resulted in prying the two cantilevers apart. However, this behavior was not as extreme as it was for the single-rod case; the lateral reaction forces were not as high. 

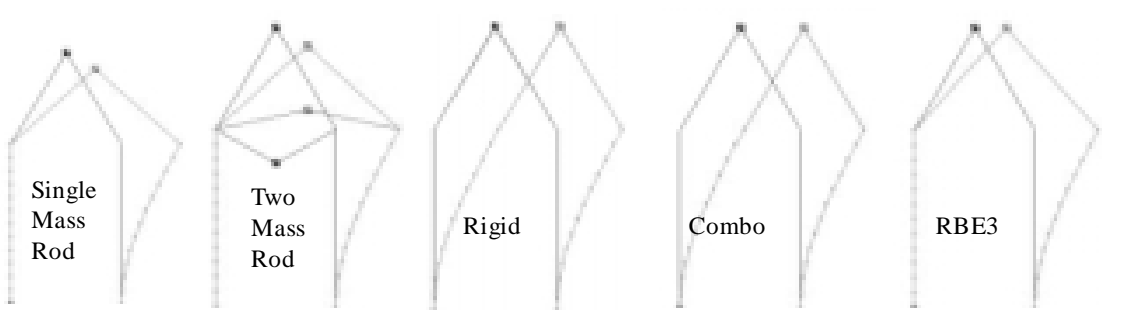

Fig. 9. Static analysis deformation plots.

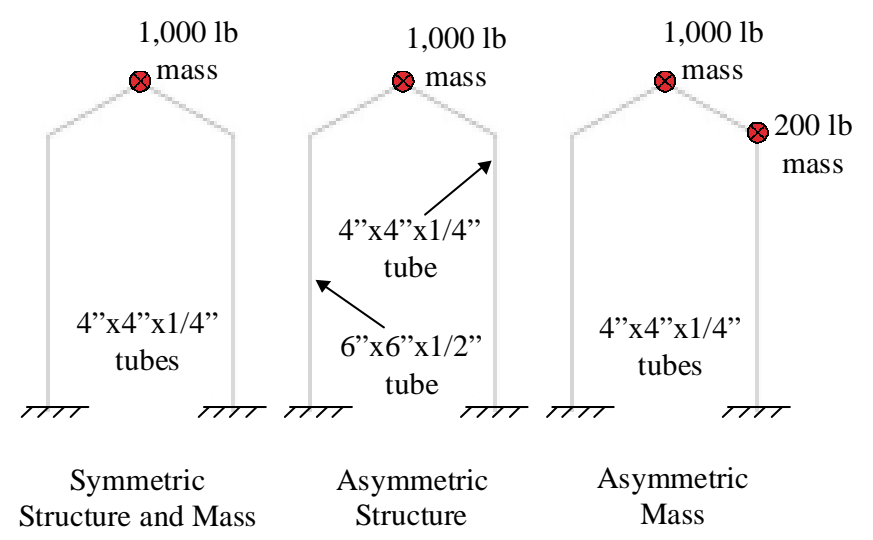

Fig. 10. Shock analysis models.

Table 2

Shock analysis results for rod cases; Symmetric structure and mass

\begin{tabular}{|c|c|c|c|c|c|c|c|c|c|}
\hline \multirow[t]{3}{*}{ Case } & \multirow[t]{3}{*}{ Mode } & \multirow[t]{3}{*}{ Freq., $\mathrm{Hz}$} & \multirow[t]{3}{*}{ Mass Part., $\%$} & \multicolumn{6}{|c|}{ Modal reaction forces } \\
\hline & & & & Left leg & & & Right leg & & \\
\hline & & & & $\mathrm{Fx}, \mathrm{lb}$ & Fy, lb & $\mathrm{Mz}$, in-lb & $\mathrm{Fx}, \mathrm{lb}$ & Fy, lb & $\mathrm{Mz}$, in-lb \\
\hline Single mass & 1 & 3.7 & $100 \mathrm{~V}$ & $-5,808$ & $-3,353$ & 418,200 & 5,808 & $-3,353$ & $-418,200$ \\
\hline Single mass & 2 & 6.4 & $100 \mathrm{~B}$ & $-3,000$ & $-1,732$ & 216,000 & $-3,000$ & 1,732 & 216,000 \\
\hline Double mass & 1 & 5.0 & $20 \mathrm{~V}$ & 2,754 & -955 & $-198,310$ & $-2,754$ & -955 & 198,310 \\
\hline Double mass & 2 & 6.4 & $100 \mathrm{~B}$ & $-3,000$ & $-1,735$ & 216,000 & $-3,000$ & 1,735 & 216,000 \\
\hline Double mass & 3 & 151.5 & $0 \mathrm{~B}$ & N/A & N/A & N/A & N/A & N/A & N/A \\
\hline Double mass & 4 & 195.5 & $80 \mathrm{~V}$ & -40 & $-84,794$ & 2,845 & 40 & $-84,794$ & $-2,845$ \\
\hline
\end{tabular}

- The Rigid, Combo and RBE3 cases shared the same response frequencies, mode shapes and reaction forces. The vertical mode resulted in no moments or lateral forces as the Rod Cases did.

A summary of the results for the asymmetric structure model is provided in Tables 4 and 5. These tables are very similar in format to Tables 2 and 3, however the mass participation column for the Rod Cases identifies mass participation values associated with both shock directions for some of the modes. For example, an ' 81 $\mathrm{V}$ ' and a ' $19 \mathrm{~B}$ ' in the column means that mode is coupled and has a vertical mass participation of $81 \%$ and a lateral mass participation of $19 \%$. Reaction forces are computed using vertical shock motion inputs for the $81 \%$ mass participation, and lateral shock motion in- puts for the $19 \%$ mass participation. The Rigid, Combo, and RBE3 Cases do not have multiple mass participation because the vertical and bending modes are uncoupled. Corresponding mode shapes are provided in Fig. 12.

The mode shapes and reaction forces were investigated and the following observations are made:

- The single-mass Rod Case again exhibited a damaging and unrealistic vertical mode that resulted in prying apart the two cantilevers. This again resulted in lateral reaction forces and moments that were extremely high compared to the other cases, and vertical forces that were low.

- The double-mass Rod Case also resulted in a prying mode but again was not as extreme as it was 
Table 3

Shock analysis results for other cases; Symmetric structure and mass

\begin{tabular}{|c|c|c|c|c|c|c|c|c|c|}
\hline \multirow[t]{3}{*}{ Case } & \multirow[t]{3}{*}{ Mode } & \multirow[t]{3}{*}{ Freq., $\mathrm{Hz}$} & \multirow[t]{3}{*}{ Mass Part., \% } & \multicolumn{6}{|c|}{ Modal reaction forces } \\
\hline & & & & \multicolumn{3}{|c|}{ Left leg } & \multicolumn{3}{|c|}{ Right leg } \\
\hline & & & & $\mathrm{Fx}, \mathrm{lb}$ & Fy, lb & $\mathrm{Mz}$, in-lb & $\mathrm{Fx}, \mathrm{lb}$ & Fy, lb & $\mathrm{Mz}$, in-lb \\
\hline Rigid & 1 & 6.4 & $100 \mathrm{~B}$ & $-3,000$ & $-1,732$ & 216,000 & $-3,000$ & 1,732 & 216,000 \\
\hline Rigid & 2 & 174.9 & $100 \mathrm{~V}$ & 0 & $-102,140$ & 0 & 0 & $-102,140$ & 0 \\
\hline Combo & 1 & 6.4 & $100 \mathrm{~B}$ & $-3,000$ & $-1,732$ & 216,000 & $-3,000$ & 1,732 & 216,000 \\
\hline Combo & 2 & 174.9 & $100 \mathrm{~V}$ & 0 & $-102,140$ & 0 & 0 & $-102,140$ & 0 \\
\hline RBE3 & 1 & 6.4 & $100 \mathrm{~B}$ & $-3,000$ & $-1,732$ & 216,000 & $-3,000$ & 1,732 & 216,000 \\
\hline RBE3 & 2 & 174.9 & $100 \mathrm{~V}$ & 0 & $-102,140$ & 0 & 0 & $-102,140$ & 0 \\
\hline
\end{tabular}

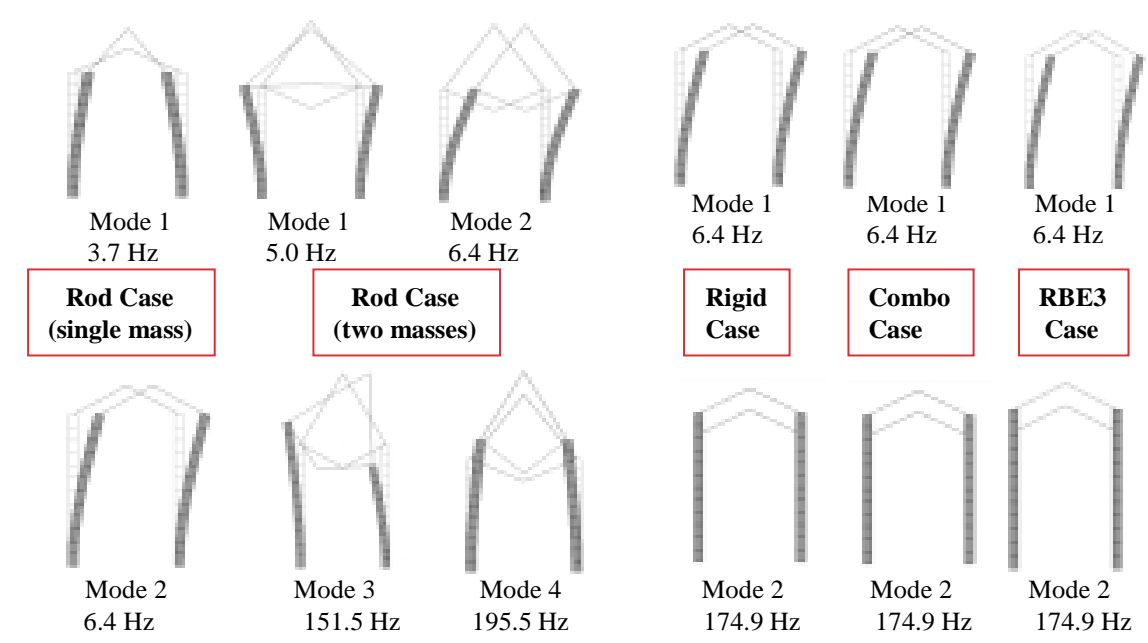

Fig. 11. Mode shapes for symmetric structure and symmetric mass.

Table 4

Shock analysis results for rod cases; Asymmetric structure

\begin{tabular}{|c|c|c|c|c|c|c|c|c|c|}
\hline \multirow[t]{3}{*}{ Case } & \multirow[t]{3}{*}{ Mode } & \multirow[t]{3}{*}{ Freq.,Hz } & \multirow[t]{3}{*}{ Mass Part., \% } & \multicolumn{6}{|c|}{ Modal reaction forces } \\
\hline & & & & Left leg & & & Right leg & & \\
\hline & & & & Fx, lb & Fy, lb & $\mathrm{Mz}$, in-lb & $\mathrm{Fx}, \mathrm{lb}$ & Fy, lb & $\mathrm{Mz}$, in-lb \\
\hline \multirow[t]{2}{*}{ Single Mass } & 1 & 4.4 & $81 \mathrm{~V}$ & 4,129 & 2,384 & $-297,320$ & $-7,311$ & 4,221 & 526,370 \\
\hline & & & $19 \mathrm{~B}$ & 1,467 & 847 & $-105,590$ & $-2,596$ & 1,499 & 186,930 \\
\hline \multirow[t]{2}{*}{ Single Mass } & 2 & 13.4 & $19 \mathrm{~V}$ & $-9,218$ & $-5,322$ & 663,670 & -833 & 481 & 59,985 \\
\hline & & & $81 \mathrm{~B}$ & $-4,467$ & $-2,579$ & 321,590 & -404 & 233 & 29,066 \\
\hline \multirow[t]{2}{*}{ Double Mass } & 1 & 5.5 & $13 \mathrm{~V}$ & 933 & $-1,360$ & $-67,144$ & $-3,178$ & -64 & 228,780 \\
\hline & & & $33 \mathrm{~B}$ & 828 & $-1,208$ & $-59,597$ & $-2,820$ & -57 & 203,070 \\
\hline \multirow[t]{2}{*}{ Double Mass } & 2 & 14.4 & $7 \mathrm{~V}$ & $-5,666$ & -784 & 407,930 & -266 & 2,667 & 19,141 \\
\hline & & & $67 \mathrm{~B}$ & $-3,828$ & -530 & 275,590 & -180 & 1,802 & 12,931 \\
\hline Double Mass & 3 & 166.9 & $21 \mathrm{~V}$ & -322 & 28,913 & 23,163 & -19 & $-79,747$ & 1,341 \\
\hline Double Mass & 4 & 304.0 & $60 \mathrm{~V}$ & -17 & $-115,270$ & 1,235 & 22 & $-14,249$ & $-1,599$ \\
\hline
\end{tabular}

for the single-mass Rod Case; the lateral reaction forces were not as high.

- The Rigid and Combo Case results were the same for both modes.

- The vertical mode shapes for the Rigid, Combo and RBE3 cases all included some degree of lateral response. For the Rigid and Combo representations, the lateral deflections of the beams were attributed to the motions of the beam tops being constrained to move together. Since one beam can deflect a greater distance than the other, the lateral motions of the beams were necessary to maintain the same distance between these points. The lateral response for the RBE3 representation was caused by a similar, but more flexible relationship among the motions of the tops of the beams and the component mass (dependent node).

A summary of the results for the asymmetric mass 
Table 5

Shock analysis results for other cases; Asymmetric structure

\begin{tabular}{|c|c|c|c|c|c|c|c|c|c|}
\hline \multirow[t]{3}{*}{ Case } & \multirow[t]{3}{*}{ Mode } & \multirow[t]{3}{*}{ Freq., $\mathrm{Hz}$} & \multirow[t]{3}{*}{ Mass Part., \% } & \multicolumn{6}{|c|}{ Modal reaction forces } \\
\hline & & & & Left leg & & & Right leg & & \\
\hline & & & & $\mathrm{Fx}, \mathrm{lb}$ & Fy, lb & $\mathrm{Mz}$, in-lb & $\mathrm{Fx}, \mathrm{lb}$ & Fy, lb & $\mathrm{Mz}$, in-lb \\
\hline Rigid & 1 & 12.2 & $100 \mathrm{~B}$ & $-5,173$ & $-1,729$ & 372,440 & -827 & 1,735 & 59,564 \\
\hline Rigid & 2 & 213.6 & $100 \mathrm{~V}$ & -164 & $-102,200$ & 11,788 & -26 & $-102,090$ & 1,885 \\
\hline Combo & 1 & 12.2 & $100 \mathrm{~B}$ & $-5,173$ & $-1,729$ & 372,440 & -827 & 1,735 & 59,564 \\
\hline Combo & 2 & 213.6 & $100 \mathrm{~V}$ & -164 & $-102,200$ & 11,788 & -26 & $-102,090$ & 1,885 \\
\hline RBE3 & 1 & 8.4 & $100 \mathrm{~B}$ & $-3,000$ & $-1,731$ & 216,000 & $-3,000$ & 1,733 & 216,000 \\
\hline RBE3 & 2 & 213.6 & $100 \mathrm{~V}$ & -45 & $-102,120$ & 3,247 & -45 & $-102,120$ & 3,247 \\
\hline
\end{tabular}

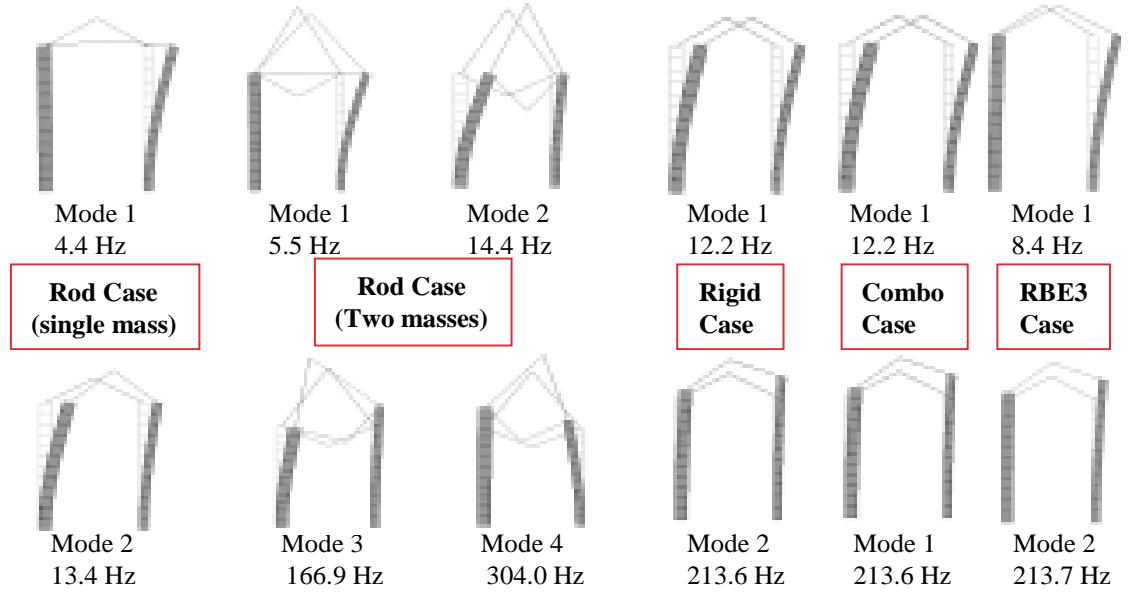

Fig. 12. Mode shapes for asymmetric structure and symmetric mass.

model is provided in Tables 6,7 , and 8 . These tables are very similar in format to Tables 4 and 5 . Corresponding mode shapes are provided in Fig. 13 and 14.

The mode shapes and reaction forces were investigated and the following observations are made:

- The single-mass Rod Case again exhibited a damaging and unrealistic vertical mode that resulted in prying apart the two cantilevers. This again resulted in lateral reaction forces and moments that were extremely high compared to the other cases, and vertical forces that were low.

- The double-mass Rod Case again resulted in a prying vertical mode. The reaction moments were high relative to those of the other vertical modes.

- The Rigid and Combo Case results were approximately the same for all modes.

- The second bending mode for the RBE3 Case was characterized by the out-of-phase lateral motion between the two mass points, but more apparently, between the tops of the beams. This mode did not meet the requirements for selection because the mass participation was insignificant.

- The second vertical mode for the RBE3 Case was characterized by the out-of-phase vertical motion between the two mass points, but more apparently, between the tops of the beams. This mode also included some lateral motion of the left leg. This response can be viewed as sensible in light of the RBE3's more flexible implementation of the Rigid Case's rigid relationship between the motions of the tops of the beams and the component mass (dependent node). This mode did not meet the requirements for selection because the mass participation was insignificant.

\section{Concluding remarks}

The observations listed above yield no definite conclusion that highlights one component representation as the correct model to use in all cases. In fact, the observations confirm the original notion that one must give careful consideration for the system being modeled, the expected modal characteristics, and compliance with NAVSEA 0908-LP-000-3010, Rev. 1. However, some brief summary observations are relevant:

- Single-mass rod element representations should be avoided when the in-plane stiffness of the foun- 
Table 6

Shock analysis results for single mass rod cases; Asymmetric mass

\begin{tabular}{lccclccccc}
\hline Case & Mode & Freq., Hz & Mass Part., \% & Modal reaction forces & & \\
\hline & & & & Left leg & & & Right leg \\
& & & & Fx, lb & Fy, lb & Mz, in-lb & Fx, lb & Fy, lb & Mz, in-lb \\
Single Mass & 1 & 3.6 & $77 \mathrm{~V}$ & $-5,029$ & $-2,094$ & 362,100 & 6,134 & $-3,102$ & $-441,640$ \\
& & & $3 \mathrm{~B}$ & -853 & -493 & 61,415 & 1,040 & -526 & $-74,906$ \\
Single Mass & 2 & 5.9 & $2 \mathrm{~V}$ & $-1,071$ & -618 & 77,124 & -878 & 336 & 63,245 \\
& & & $97 \mathrm{~B}$ & $-3,853$ & $-2,225$ & 277,420 & $-3,160$ & 1,207 & 227,490 \\
Single Mass & 3 & 246.1 & $21 \mathrm{~V}$ & 5 & 3 & -351 & -37 & $-59,747$ & 2,637 \\
\hline
\end{tabular}

Table 7

Shock analysis results for double-mass rod cases; Asymmetric mass

\begin{tabular}{|c|c|c|c|c|c|c|c|c|c|}
\hline \multirow[t]{3}{*}{ Case } & \multirow[t]{3}{*}{ Mode } & \multirow[t]{3}{*}{ Freq., $\mathrm{Hz}$} & \multirow[t]{3}{*}{ Mass Part., \% } & \multicolumn{6}{|c|}{ Modal reaction forces } \\
\hline & & & & Left Leg & & & Right Leg & & \\
\hline & & & & $\mathrm{Fx}, \mathrm{lb}$ & Fy, lb & $\mathrm{Mz}$, in-lb & Fx, lb & Fy, lb & $\mathrm{Mz}$, in-lb \\
\hline Double Mass & 1 & 4.6 & $13 \mathrm{~V}$ & 1,728 & -917 & $-124,400$ & $-3,052$ & -519 & 219,710 \\
\hline Double Mass & 2 & 6.0 & $2 \mathrm{~V}$ & $-1,109$ & -317 & 79,857 & -628 & 561 & 45,215 \\
\hline & & & $89 \mathrm{~B}$ & $-4,072$ & $-1,165$ & 293,220 & $-2,306$ & 2,058 & 166,020 \\
\hline Double Mass & 3 & 137.0 & $10 \mathrm{~V}$ & -44 & 36,927 & 3,191 & -25 & $-65,208$ & 1,807 \\
\hline
\end{tabular}

Table 8

Shock analysis results for other cases; Asymmetric mass

\begin{tabular}{|c|c|c|c|c|c|c|c|c|c|}
\hline Case & Mode & Freq., $\mathrm{Hz}$ & Mass Part., \% & \multicolumn{6}{|c|}{ Modal reaction forces } \\
\hline & & & & Left Leg & & & Right Leg & & \\
\hline & & & & $\mathrm{Fx}, \mathrm{lb}$ & Fy, lb & $\mathrm{Mz}$, in-lb & $\mathrm{Fx}, \mathrm{lb}$ & Fy, lb & $\mathrm{Mz}$, in-lb \\
\hline Rigid & 1 & 5.9 & $100 \mathrm{~B}$ & $-3,600$ & $-1,732$ & 259,200 & $-3,600$ & 1.733 & 259,200 \\
\hline Rigid & 2 & 157.0 & $99.3 \mathrm{~V}$ & -15.5 & $-93,723$ & 1,113 & -15.5 & $-141,450$ & 1.113 \\
\hline Rigid & 3 & 377.3 & $0.7 \mathrm{~V}$ & 3.4 & $-6,287$ & -243.4 & 3.4 & $-4,165$ & -243.5 \\
\hline Combo & 1 & 5.9 & $100 \mathrm{~B}$ & $-3,600$ & $-1,732$ & 259,200 & $-3,600$ & 1,733 & 259,200 \\
\hline Combo & 2 & 157.0 & $99.3 \mathrm{~V}$ & -15.5 & $-93,723$ & 1.113 & -15.5 & $-141,450$ & 1.113 \\
\hline Combo & 3 & 377.3 & $0.7 \mathrm{~V}$ & 3.4 & $-6,287$ & -243.4 & 3.4 & $-4,165$ & -243.5 \\
\hline RBE3 & 1 & 5.8 & $99.5 \mathrm{~B}$ & $-2,893$ & $-1,670$ & 208,300 & $-4,273$ & 1,671 & 307,620 \\
\hline RBE3 & 2 & 16.0 & $0.5 \mathrm{~B}$ & N/A & N/A & N/A & N/A & N/A & N/A \\
\hline RBE3 & 3 & 157.2 & $99.5 \mathrm{~V}$ & -35.4 & $-95,134$ & 2,552 & 0 & -140.460 & -11 \\
\hline RBE3 & 4 & 435.4 & $0.5 \mathrm{~V}$ & N/A & N/A & N/A & N/A & N/A & N/A \\
\hline
\end{tabular}

dation is relatively low. In general, they are reasonable when the component mass point is located well above the bolting plane and the foundation's in-plane stiffness is somewhat greater than its vertical stiffness.

- Double-mass rod element representations should be avoided when the component mass is not in or close to the bolting plane, and when the foundation structural stiffness and mass are not reasonably balanced (symmetric).

- Pinned rigid frames transfer load through the component and can over-constrain the foundation. Their use is reasonable when information dependent on rotations of the component mass point is important or when the constraint that they add to the model is justified.

- The combination of rods and an RBE3 behaves essentially the same as a pinned rigid frame in two- dimensional problems. For three-dimensional analyses, some independent motion of the rods is permitted while the element's behavior approximates that of the rigid frame in an average sense.

- The RBE3 element is acceptable for all cases herein.

Some of the cases comply with the 3010 manual, yet produce mode shapes and reaction forces that are unrealistic. If a component were stiff, then one would expect the models herein to behave such that the cantilevers displace as if the tops were tied together. However, achieving these modes require component representations that are rigid and violate the fundamental principle of structural constraint mentioned in the 3010 manual.

The observations listed herein, and knowledge about the various component representations, were used to 

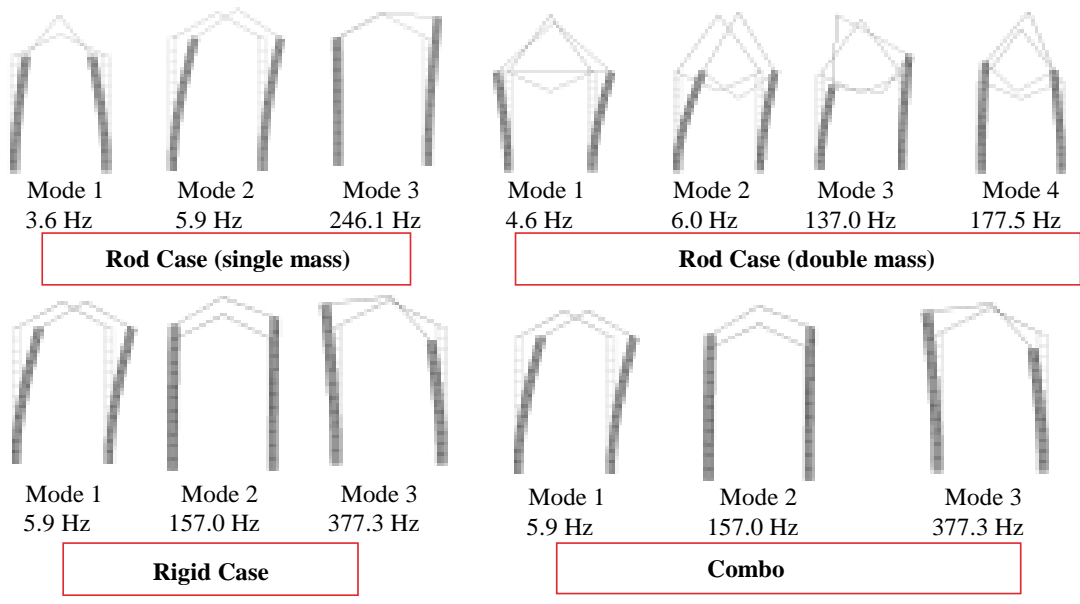

Fig. 13. Mode shapes for symmetric structure and asymmetric mass.

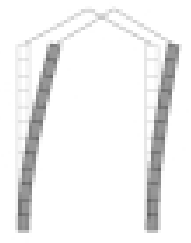

Mode 1 $5.8 \mathrm{~Hz}$

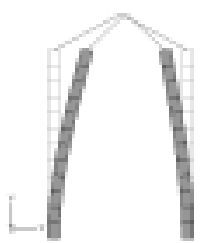

Mode 2

$16.0 \mathrm{~Hz}$

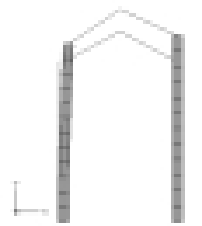

Mode 3

$157.2 \mathrm{~Hz}$

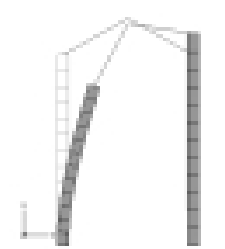

Mode 4

$435.4 \mathrm{~Hz}$

RBE3 Case

Fig. 14. Mode shapes for symmetric structure and asymmetric mass, continued.

Table 9

Shock analysis results for other cases; Asymmetric mass

\begin{tabular}{lll}
\hline Case & Advantage & Disadvantage \\
\hline Single Mass Rod & Complies with 3010 requirements. & Potential for 'prying'. Rotary inertia is unavailable. \\
Double Mass Rod & Complies with 3010 requirements. & Potential for 'prying'. Rotary inertia is unavailable. \\
Rigid & Rotary inertia is available. & Potential 3010 violation. \\
Combination & Does not redistribute mass. & Potential 3010 violation. Rotary inertia is unavailable. \\
Interpolation & $\begin{array}{l}\text { Complies with 3010 requirements. } \\
\text { tary inertia is available. }\end{array}$ & Can sometimes lead to insignificant, but unintuitive modes. \\
& & \\
\hline
\end{tabular}

compile Table 9, summarizing the advantages and disadvantages of the various modeling techniques to represent the component.

The comments in Table 9 are intended to be generic and widely applicable, however, one must not be quick to assume that these comments absolutely apply to their system model.

\section{References}

[1] NAVSEA 0908-LP-000-3010, Rev. 1, Shock Design Criteria for Surface Ships, Prepared by Supervisor of Shipbuilding, Conversion and Repair, USN, Brooklyn under the direction of Naval Sea Systems Command, September 1995.

[2] The MacNeal-Schwendler Corporation, MSC NASTRAN, Version 70.0.0, dated 1997.

[3] Hibbitt, Karlsson, and Sorensen, Inc., ABAQUS Standard, Version 5.8, dated 1998. 

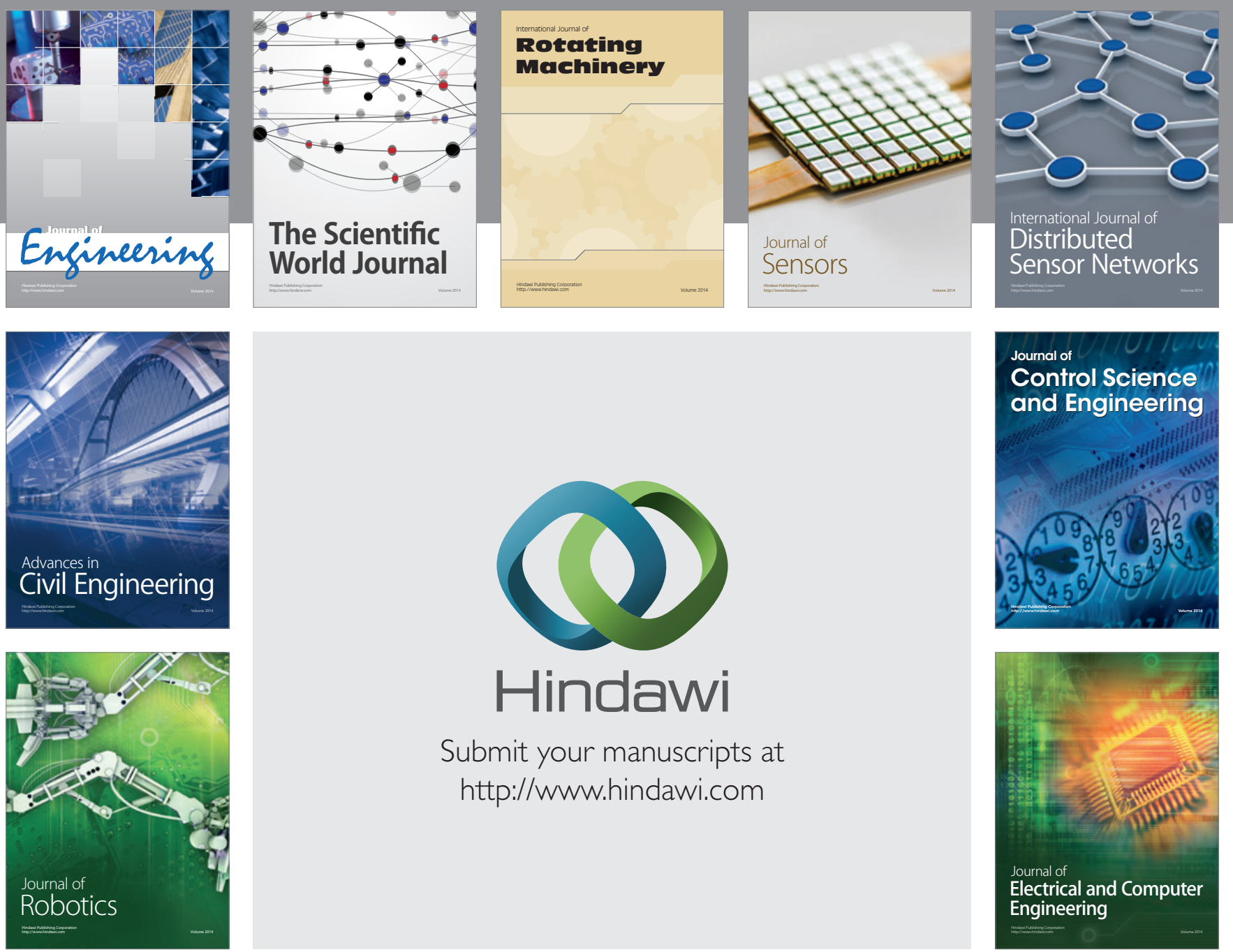

Submit your manuscripts at

http://www.hindawi.com
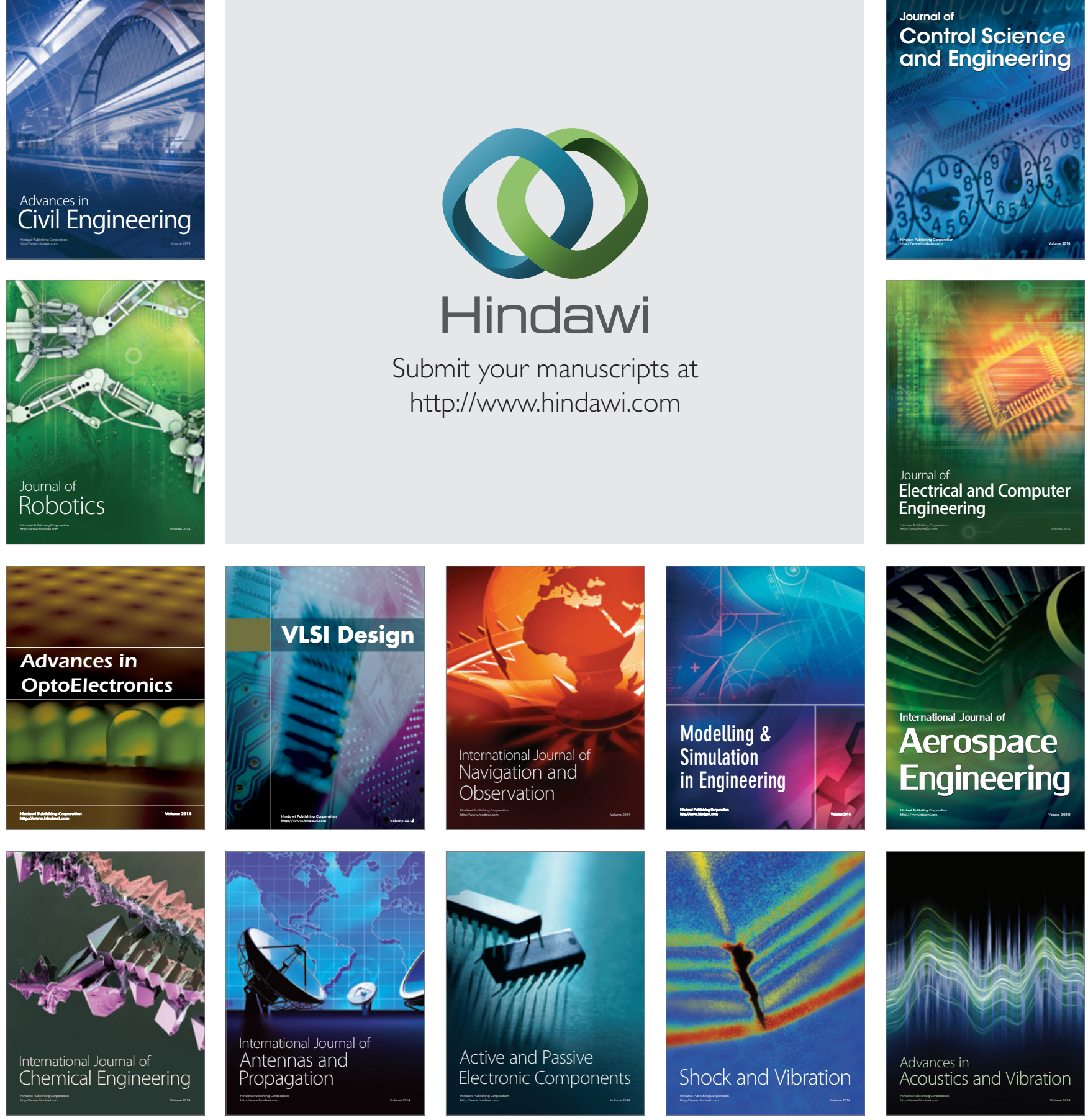